\title{
FEMTOSECOND ELECTRON AND X-RAY GENERATION BY LASER AND PLASMA-BASED SOURCES
}

\author{
E. ESAREY AND W.P. LEEMANS \\ Center for Beam Physics, Accelerator and Fusion Research Division \\ Ernest Orlando Lawrence Berkeley National Laboratory \\ One Cyclotron Road, University of California, Berkeley CA 94720
}

\begin{abstract}
The generation of ultra-short x-rays by Thomson scattering intense laser pulses from electron beams is discussed, including recent experimental results and methods for enhancing the x-ray flux. A high flux of x-rays in a femtosecond pulse requires the generation of femtosecond electron bunches and a head-on Thomson scattering geometry. The generation of ultrashort electron bunches in a plasmabased accelerator with an injection technique that uses two colliding laser pulses is discussed. Simulations indicate the bunches as short as a few fs can be produced. Conversion of the fs electron pulse to a fs x-ray pulse can be accomplished by Bremsstrahlung or Thomson scattering.
\end{abstract}

\section{Introduction}

The use of femtosecond x-ray pulses is becoming an increasingly important tool in the study of ultrafast structural dynamics in crystals. ${ }^{1}$ Optical wavelengths have been used in the past to provide indirect information on laser excited ultra-fast disordering in materials such as $\mathrm{Si}$, GaAs and InSb. ${ }^{2}$ However, since these photons interact primarily with outer shell electrons, only an indirect measure of the material structure can be obtained. X-rays, on the other hand, can provide a direct measurement of the crystal structure through measurement of the diffraction pattern.

To enable time-resolved measurement of structural changes requires the production of ultra-short x-ray pulses. The typical time scale for lattice changes in a crystal is related to the characteristic vibrational time, which is on the order of 100 fs. Furthermore, since ultra-short laser pulses are typically used to initiate the material changes, synchronization between laser and x-ray source is an essential requirement to allow pump-probe type experiments.

In this paper, we discuss two methods for the generation of fs x-ray pulses through the interaction of a relativistic electron beam with an intense laser pulse. ${ }^{3-6}$ The first method employs Thomson scattering an intense laser pulse off a relativistic electron beam..$^{7-13}$ The second method is a technique for the production of ultra-short electron bunches (and hence ultra-short x-ray pulses) by a laser-plasma injector, in which low emittance electron bunches are expected to be produced through the use of colliding laser pulses. ${ }^{14-17}$ 
These laser-electron beam sources offer some unique advantages: (1) they can generate hard x-rays with substantial flux in a highly directed beam; (2) the source parameters such as photon energy, brightness, and bandwidth, are controllable through electron beam and laser parameters; (3) the pulse length is controllable through the laser pulse length, electron bunch length, and interaction geometry; and (4) the methods provide perfect synchronization between the laser and x-ray pulses.

In Sec. 2 we will discuss generation of fs x-rays by Thomson scattering a high power laser beam off a relativistic electron beam, including a brief review of the theory, and recent experiments at LBNL, ${ }^{7-10}$ Brookhaven National Laboratory (BNL) ${ }^{11}$ and Thomas Jefferson National Laboratory (TJNL). ${ }^{12}$ Methods for enhancing the flux and brightness of future sources are discussed. Section 3 covers the use of laser-plasma based accelerators and the colliding pulse method for production of ultra-short electron bunches. We conclude in Sec. 4 with a brief discussion of ultra-short x-ray sources that rely on the interaction of laser pulses with electron bunches produced by linacs and novel laser-plasma injectors.

\section{Ultra-short x-ray pulses from Thomson scattering}

\subsection{Properties of Thomson scattered x-rays}

Scattering of laser light off an electron beam (e-beam) was proposed ${ }^{18,19}$ and used to diagnose low energy e-beams ${ }^{20}$ in the early sixties. More recently, with the development of intense, short-pulse lasers based on the chirped-pulse amplification technique ${ }^{21}$ and with high power free electron lasers (FEL), Thomson scattering is being considered as a radiation source capable of producing directed, bright, short pulses of tunable radiation as well as a means for diagnosing electron beams. Effectively, the laser field acts as an electromagnetic undulator for the electron beam. Since the undulator period is determined by the laser wavelength, ultra-short wavelength radiation (x-rays or $\gamma$-rays) can be generated using electron beams of modest energies. Conventional solid state or gas laser systems which deliver multi-TW peak power, $100 \mathrm{fs}$ pulses at a repetition rate of $10 \mathrm{~Hz}$ are becoming readily available as well as FEL's with high average power. Combined with the development of high current (>100 A peak current), low emittance electron beam sources, these laser systems allow development of high brightness x-ray and $\gamma$-ray sources, ${ }^{22}$ as well as diagnostics and control of relativistic particle beams. ${ }^{8,23}$ Experiments on non-linear scattering of a terawatt laser pulse off a $50 \mathrm{GeV}$ electron beam have been conducted at $\mathrm{SLAC}^{24}$ and generation of VUV-pulses is being studied at 
NRL. ${ }^{25}$ This paper only discusses the generation of fs x-ray pulses.

The Thomson scattered x-ray wavelength, $\lambda_{x}$, is given by

$$
\lambda_{x}=\frac{\lambda_{L}}{2 \gamma^{2}} \frac{\left(1+\gamma^{2} \theta^{2}+K_{L}^{2} / 2\right)}{(1-\cos \psi)} N_{H},
$$

assuming $\gamma^{2} \gg\left(1+K_{L}^{2} / 2\right), \theta^{2} \ll 1$, and linear polarization, where $\gamma$ is the Lorentz factor, $N_{H}$ is the harmonic number, $\lambda_{L}$ is the laser wavelength, $\psi$ is the angle of interaction between the laser and each electron (e.g., $\psi=180^{\circ}$ for head-on scattering), $\theta$ is the observation angle (relative to the initial electron trajectory), and $K_{L}=e E_{L} \lambda_{L} / 2 \pi m c^{2}$ is the normalized vector potential of the peak laser field, $E_{L}$. Here $e$ and $m$ are the charge and rest mass of an electron, respectively, and $c$ is the speed of light. In terms of the laser intensity, $I_{L}, K_{L}^{2} \simeq 7.3 \times 10^{-19} \lambda_{L}^{2}[\mu \mathrm{m}] I_{L}\left[\mathrm{~W} / \mathrm{cm}^{2}\right]$. In the low intensity limit $K_{L}^{2} \ll 1$, radiation is scattered only at the fundamental $N_{H}=1$. In the nonlinear limit $K_{L}^{2} \gg 1$, numerous harmonics are generated. ${ }^{4,6}$ This results in a near continuum of scattered radiation with harmonics extending out to a critical harmonic number (e.g., $N_{c r}=3 K_{L}^{3} / 4$ for $\psi=180^{\circ}$ ), beyond which the intensity of the scattered radiation rapidly decreases. ${ }^{4,6}$ Note that here, because the laser photon energy in the electron beam rest frame $\left(\gamma \hbar \omega_{L}\right)$ is much less than the electron rest energy $\left(m c^{2}\right)$, Compton recoil can indeed be neglected. Also, for relativistic electron beams, the x-ray flux is strongly peaked in the forward direction with a radiation cone opening angle of $1 / \gamma$.

In the limit $K_{L}^{2} \ll 1$, the number of x-rays scatter per electron, at all angles and frequencies, is $n_{x s}=(\pi / 3) \alpha K_{L}^{2} M_{L}^{*}$, where $M_{L}^{*}$ is the effective number of laser periods with which the electron interacts and $\alpha=1 / 137$ is the fine structure constant. The total number of x-rays, $n_{x}$, scattered by a single bunch of $N_{b}$ electrons into a narrow bandwidth $\delta \omega / \omega \ll 1$ is ${ }^{3-6,9}$

$$
n_{x}=2 \pi \alpha K_{L}^{2} M_{L} N_{b} F_{G} F_{\text {coll }} \delta \omega / \omega,
$$

where $M_{L}$ is the total number of optical cycles in the laser pulse (number of wiggler periods) and $F_{G}$ is a factor that depends on the particular geometry of the interaction. For head-on scattering, $F_{G} \simeq 1$ assuming $\sigma_{b}<\sigma_{L} \leq 4 Z_{R} \leq \beta$ and $r_{L} \geq r_{b}$, where $\sigma_{b}\left(\sigma_{L}\right)$ is the electron bunch (laser pulse) length, $r_{b}\left(r_{L}\right)$ is the electron bunch (laser pulse) radius, $Z_{R}=\pi r_{L}^{2} / \lambda_{L}$ is the laser Rayleigh length and $\beta$ is the beta-function of the electron beam. For $90^{\circ}$ scattering, $F_{G} \simeq \sigma_{L} / \sigma_{b}$, assuming $\sigma_{L} \leq 2 r_{b} \leq \sigma_{b} \leq \beta$ and $r_{L} \geq r_{b} . F_{\text {coll }}$ is a factor determined by the collection angle of the x-ray optics, $\theta_{\text {det }}$ and is given by $F_{\text {coll }}=\theta_{\text {det }}^{2} /\left(\theta_{\text {det }}^{2}+\theta_{T}^{2}\right)$, where $\theta_{T}^{2}=\theta_{b}^{2}+\theta_{\text {int }}^{2}+\theta_{\delta}^{2}$ and $\theta_{b}=\epsilon_{N} /\left(\gamma \sigma_{r}\right)$ is the electron beam rms opening angle, $\theta_{\text {int }}=1 /\left(\gamma^{2} M_{L}\right)^{1 / 2}$ is the intrinsic xray beam opening angle and $\theta_{\delta}=(\delta \omega / \omega)^{1 / 2} / \gamma$ is the contribution from the 
finite bandwidth. Here $\epsilon_{N}=\gamma \epsilon$ is the normalized emittance of the electron beam, with $\epsilon$ the rms unnormalized emittance and $\sigma_{r} \simeq 1.2 r_{b}$. X-rays in the bandwidth $\delta \omega / \omega$ will be confined to a cone-angle about the direction of the electron given by $\theta_{c} \simeq \theta_{T}$. A limiting collection angle $\theta_{\text {det }}$ of a few mrads is typical, since in practice grazing incidence optics are used.

The source brightness is then given by

$$
B_{x}=n_{x}\left(2 \pi \sigma_{r} \sigma_{\theta}\right)^{-2}
$$

where $\sigma_{r} \approx r_{b}$ and $\sigma_{\theta} \approx \theta_{T}$ are the rms source size and opening angle.

The scattered x-ray energy spectrum can be calculated by accounting for the spatial profile of the electron beam via a convolution of the single electron spectrum with the angular distribution function of the electron beam and integrating over all azimuthal angles. This has been done in detail for the case of $90^{\circ}$ scattering in the linear limit $K_{L}^{2} \ll 1$. $^{9}$

Generation of fs duration pulses through Thomson scattering requires using fs laser pulses combined with either the use of a scattering geometry in which the interaction time between the photon and electron beams is limited, or development of very short electron bunches. ${ }^{26-29}$ In a backscattering geometry, the duration of the scattered beam is typically determined by the length of the electron beam. ${ }^{3,4}$ In the $90^{0}$ geometry where both beams are tightly focused,$^{5-9}$ the interaction time between the electron beam and laser beam is typically limited to the transverse rather than the longitudinal transit time of the laser pulse across the electron beam. Ultra-short x-ray pulses can then be generated from long electron bunches.

\section{2 $90^{\circ}$ Thomson scattering experiments at $L B N L$}

In the first demonstration of $90^{\circ}$ Thomson scattering, ${ }^{7-9} 300$ fs (FWHM) xrays were produced using a $0.5 \mathrm{TW}$ laser pulse $\left(\sigma_{L} \approx 60 \mathrm{fs}, \lambda_{L}=0.8 \mu \mathrm{m}, 40\right.$ $\mathrm{mJ} /$ pulse) with a $50 \mathrm{MeV}$ electron bunch $\left(N_{b} \approx 10^{10}, \sigma_{b} \approx 15 \mathrm{ps}\right)$, focused to a spot size of $90 \mu \mathrm{m} \mathrm{FWHM}\left(\sigma_{r}=1.2 r_{b}=38 \mu \mathrm{m}\right)$. The experiment was carried out at the Beam Test Facility ${ }^{30}$ in which the electron bunches were transported to an interaction chamber where they were focused and scattered against the laser beam. After the interaction chamber, a $60^{\circ}$ bend magnet deflected the electron beam onto a beam dump, away from the forward scattered x-rays. A $75 \mathrm{~cm}$ radius of curvature mirror was used to focus the S-polarized amplified laser pulses to about a $30 \mathrm{~mm}$ diameter spot at the interaction point (IP), measured by a charge coupled device (CCD) camera at an equivalent image plane outside the vacuum chamber. To measure the spot size (and position) of the electron beam at the IP, an image of the electron beam was obtained 
by relaying optical transition radiation (OTR) from a foil onto a 16 bit CCD camera or optical streak camera using a small f-number telescope. ${ }^{9}$

The source characteristics measured in the experiments were the total flux, spatial profile of the x-ray beam and the spectrum of the radiation for different observation angles. The total flux and profile of the x-ray beam were measured using a phosphor screen, located $80 \mathrm{~cm}$ from the IP. Visible photons from the screen were imaged onto a 2 -D $(512 \times 512), 16$ bit CCD camera. The detection system had a full collection angle of $25 \mathrm{mrad}$.

From these x-ray beam profiles, the electron beam divergence of the temporal slice with which the laser pulse interacts can be obtained. For example, by fitting the data and comparing with theory, an electron beam divergence of $\sigma_{\theta_{x}}\left(\sigma_{\theta_{y}}\right)=6.3 \pm 0.2(3.9 \pm 0.2) \mathrm{mrad}$ was found. The difference between $\sigma_{\theta_{x}}$ and $\sigma_{\theta_{y}}$ is due to a combination of the electron beam being focused astigmatically at the IP, resulting in a tilted phase space ellipse $\left(y, y^{\prime}\right)$, and a laser spot size much smaller than the vertical electron beam size. The ellipticity of the image results from astigmatism in the electron beam focusing due to the use of quadrupole focusing magnets. The smaller horizontal than vertical spot size of the electron beam, causes a stronger electron beam divergence in the horizontal (and therefore stronger x-ray beam divergence) than in the vertical direction. The total number of photons within the collection angle was typically $5 \times 10^{4}$ photons.

The x-ray spectra at observation angles $\theta=0,5$, and $10 \mathrm{mrad}$ were measured with a liquid nitrogen cooled Ge-detector, located $2 \mathrm{~m}$ from the interaction point. At larger observation angles, the low energy side of the spectrum broadened because of the combined effects of the electron beam divergence and the correlation between emission angle and wavelength. In addition, there is a corresponding reduction in the measured flux. Comparing the measured spectra with theory provides an estimate of the effective angular divergence of the electron beam on the order of $3.5-4 \mathrm{mrad}$. The peak amplitudes of the spectra at 5 and $10 \mathrm{mrad}$ are lower by factors of 0.47 and 0.06 , respectively, than the peak of the 0 mrad spectrum. Because the energy spread of the electron beam is small $(\Delta \gamma / \gamma \leq 0.5 \%)$, its contribution to the x-ray spectrum is negligible. The effect of the finite laser bandwidth ( $25 \mathrm{~nm}$ FWHM) is included and results in the spectrum having a softer roll-off on the high energy side. The spectral width was about $8 \%$ with the main contribution coming from the electron beam divergence. The large beam divergence is a result of tightly focusing the electron beam, which has a normalized emittance $\epsilon_{N}=30$ $\mathrm{mm}$-mrad. The electron beam size in this work represents a compromise of $\mathrm{x}$-ray pulse duration, spectral bandwidth, and beam divergence.

Based on these measurements, the peak and average flux were on the 
order of $6 \times 10^{2} \mathrm{ph} / \mathrm{s} / 0.1 \%$ BW, which is in agreement with Eq. (2), and $1.2 \times 10^{3} \mathrm{ph} / \mathrm{s} / 0.1 \% \mathrm{BW}(2 \mathrm{~Hz}$ repetition rate of the linac), respectively. The peak brightness was therefore $3.4 \times 10^{3} \mathrm{ph} / \mathrm{s} / 0.1 \% \mathrm{BW} / \mathrm{mm}^{2}-\mathrm{mrad}^{2}$, where we used an rms-spot size of $38 \mu \mathrm{m}$ and an rms-divergence of $8 \mathrm{mrad}$. The $90^{\circ}$ Thomson scattering source at LBNL has recently been used to study ultrafast structural dynamics in InSb by time-resolved x-ray diffraction. ${ }^{10}$

\subsection{Flux Enhancement Methods}

The number of x-rays and peak brightness of the $90^{\circ}$ Thomson scattering source experiments at LBNL was in part limited by the fact that the laser beam only interacted with about a 100 fs long electron beam slice (or $0.3 \%$ of all the available electrons), as well as the relatively high transverse emittance of the electron beam used in the experiment. There are several immediate opportunities for enhancing the flux by several orders of magnitude.

For diffraction experiments, x-ray energies on the order of 5-10 keV are desirable. Using Eqs. (2)-(3) an expression for the number of x-rays produced through $90^{\circ}$ Thomson scattering can be obtained under the following assumptions: the collection angle of the x-ray optics is smaller than $1 / \gamma$, and the beam emittance is sufficiently small to result in a negligible increase in x-ray beam divergence and bandwidth, i.e., $\epsilon_{N} \leq \sigma_{r} / \sqrt{M_{L}}$. Furthermore, the electron beam radius, $r_{b}$, laser pulse radius, $r_{L}$, and and laser pulse length, $\sigma_{L}$, are matched for optimum interaction, i.e., $\sigma_{L} \approx 2 r_{L} \approx 2 r_{b} \ll \sigma_{b}$ and the wiggler parameter $K_{L}^{2} \ll 1$. The x-ray pulse duration can be estimated from $c \tau_{x} \approx \sigma_{L}+2 r_{b}$. The collected number of x-ray photons per pulse is then

$$
N_{x, 90^{\circ}}=8 \alpha N_{b} \frac{U_{L}}{m c^{2}} \frac{r_{e}}{Z_{R}} \frac{\sigma_{L}}{\sigma_{b}} \frac{\delta \omega}{\omega} F_{\text {coll }} .
$$

The brightness can be written as

$$
B_{x, 90^{\circ}}=f_{r e p} \frac{\alpha}{\pi^{2}} N_{b} \frac{U_{L}}{m c^{2}} \frac{\sigma_{L}}{\sigma_{b}} \frac{r_{e}}{Z_{R}} \frac{\lambda_{L}}{\epsilon_{N}^{2} \lambda_{X}} \frac{\delta \omega}{\omega} \frac{\theta_{b}^{2}}{\theta_{T}^{2}},
$$

where $f_{\text {rep }}$ is the repetition rate (pulses/s) and $\lambda_{L}=2 \gamma^{2} \lambda_{X}$. Note that the usual expression for brightness utilizes a spectral bandwidth $\delta \omega / \omega=0.1 \%$.

As can be seen from Eq. (4) the first improvement can arise from reduction of the electron bunch duration. Significant progress on various approaches of generating fs electron bunches from photocathode driven linacs has been reported. Kung et al. ${ }^{26}$ used an alpha magnet with energy filtering to select out a $50 \mathrm{fs}(\mathrm{rms})$ slice containing about 2-4.6 $\times 10^{8}$ electrons per micro-bunch, from an electron bunch produced by a radio-frequency gun with a thermionic cathode coupled to an S-band linac. Uesaka et al. ${ }^{27}$ produced $300 \mathrm{fs}$ (rms) 
long bunches through compression in a non-isochronous magnetic transport line of $10 \mathrm{ps}$ long single electron bunches, which had a few percent timecorrelated energy chirp. Carlsten et al. ${ }^{28}$ produced less than 1 ps electron bunches $(8 \mathrm{MeV}, 1.1 \mathrm{nC})$ by compression using a four-dipole chicane magnet. Wang et al. ${ }^{29}$ generated ultrashort electron bunches (400 fs, $40 \mathrm{pC}$ ) with low energy spread $(0.15 \%)$ and normalized emittance $(0.5 \mathrm{~mm}-\mathrm{mrad})$ from a photocathode RF gun with no magnetic compression. Typically, therefore, a $1 \mathrm{nC}$ bunch can be shortened to about 1 ps.

Second, the laser pulse energy $U_{L}$ (i.e., number of photons $N_{p h}$ ) can be increased by about two orders of magnitude. High peak power $(10 \mathrm{TW}$ in $100 \mathrm{fs}$ ) laser systems can operate at $10 \mathrm{~Hz}$, and when focused to a sufficiently large spot size for $K_{L}<1$, can further increase the yield by about one to two orders of magnitude.

A third and important increase in flux and brightness can come from the use of lower emittance linacs such as photocathode driven accelerators. The rms divergence of the x-ray beam, $\sigma_{\theta_{x-\text { ray }}}$, generated via Thomson scattering can be determined from the finite divergence of the electron beam, $\sigma_{\theta_{e}}$ and the finite number of laser periods ${ }^{3-6}$

$$
\sigma_{\theta_{x-r a y}}^{2} \approx\left(\sigma_{\theta_{e}}\right)^{2}+\frac{\left(1+K_{L}^{2} / 2\right)}{2 \gamma^{2} M_{L} N_{H}} .
$$

By lowering the beam emittance, a reduction in divergence can therefore be accomplished. Also, for a given divergence angle, lower emittance implies smaller beam spot size and hence an increase in $K_{L}$. The reduction in beam divergence also can significantly reduce the spectral bandwidth.

If sub-ps electron bunches are available, a further increase in flux and brightness can be obtained using counter-propagating electron and laser beams. Expressions for number of x-rays per pulse and brightness can be obtained in a similar manner as was done for the $90^{\circ}$ case. Assuming $\sigma_{b}<\sigma_{L} \leq 4 Z_{R} \leq \beta$ and $r_{L} \geq r_{b}$, the number of x-rays per pulse is

$$
N_{x, 180^{\circ}}=8 \alpha N_{b} \frac{U_{L}}{m c^{2}} \frac{r_{e}}{Z_{R}} \frac{\delta \omega}{\omega} F_{\text {coll }},
$$

and the source brightness is

$$
B_{x, 180^{\circ}}=f_{\text {rep }} \frac{\alpha}{2 \pi^{2}} N_{b} \frac{U_{L}}{m c^{2}} \frac{r_{e}}{Z_{R}} \frac{\lambda_{L}}{\epsilon_{N}^{2} \lambda_{X}} \frac{\delta \omega}{\omega} \frac{\theta_{b}^{2}}{\theta_{T}^{2}} .
$$

For a fixed x-ray wavelength, it can be seen from Eq. (7) that the yield increases linearly with laser wavelength for the same spot size and linear with laser energy. Of course, longer laser wavelength implies operation at higher 
Table 1. Example of a $12.4 \mathrm{keV}$ x-ray source for $90^{\circ}$ Thomson scattering using a $0.8 \mu \mathrm{m}$ laser. The collection angle is assumed to be $1 \mathrm{mrad}$ and axisymmetric.

$\begin{array}{ll}\text { Laser wavelength } \lambda_{L} & 0.8 \mu \mathrm{m} \\ \text { Laser pulse energy } U_{L} & 300 \mathrm{~mJ} \\ \text { Laser pulse duration (FWHM) } \tau_{L} & 100 \mathrm{fs} \\ \text { Electron beam energy } \gamma & 70 \\ \text { Number of electrons } N_{b} & 10^{10} \\ \text { Beam radii } r_{L}=\sigma_{r} & 14 \mu \mathrm{m} \\ \text { Electron bunch length } \sigma_{b} & 140 \mu \mathrm{m} \\ \text { Normalized emittance } \epsilon_{N} & 2 \mathrm{~mm}-\mathrm{mrad} \\ \text { Bandwidth } \delta \omega / \omega & 10^{-3} \\ \text { Repetition rate } & 10 \mathrm{~Hz} \\ \text { Flux }(\mathrm{ph} / \mathrm{s} / 0.1 \% \mathrm{BW}) & 7.3 \times 10^{5} \\ \text { Brightness }\left(\mathrm{ph} / \mathrm{s} / \mathrm{mm}^{2}-\mathrm{mrad}^{2} / 0.1 \% \mathrm{BW}\right) & 8.5 \times 10^{7}\end{array}$

Table 2. Example of a $12.4 \mathrm{keV}$ x-ray source for $180^{\circ}$ Thomson scattering using a $10 \mu \mathrm{m}$ laser. The electron bunch has a duration of $100 \mathrm{fs}$ (FWHM) which determines the duration of the x-ray pulse. The peak electron beam current is the same as for Table 1. The collection angle is assumed to be 1 mrad and axisymmetric.

$\begin{array}{ll}\text { Laser wavelength } \lambda_{L} & 10 \mu \mathrm{m} \\ \text { Laser pulse energy } U_{L} & 0.3 \mathrm{~J} \\ \text { Laser pulse duration }(\mathrm{FWHM}) \tau_{L} & 3 \mathrm{ps} \\ \text { Electron beam energy } \gamma & 177 \\ \text { Number of electrons } N_{b} & 10^{9} \\ \text { Beam radii } r_{L}=2 \sigma_{r} & 30 \mu \mathrm{m} \\ \text { Electron bunch length } \sigma_{b} & 14 \mu \mathrm{m} \\ \text { Normalized emittance } \epsilon_{N} & 2 \mathrm{~mm}-\mathrm{mrad} \\ \text { Bandwidth } \delta \omega / \omega & 10^{-3} \\ \text { Repetition rate } & 10 \mathrm{~Hz} \\ \text { Flux }(\mathrm{ph} / \mathrm{s} / 0.1 \% \mathrm{BW}) & 1.1 \times 10^{7} \\ \text { Brightness }\left(\mathrm{ph} / \mathrm{s} / \mathrm{mm}^{2}-\mathrm{mrad}^{2} / 0.1 \% \mathrm{BW}\right) & 1.9 \times 10^{9}\end{array}$

electron beam energy. The brightness, Eq. (8), increases quadratically with laser wavelength for the same laser spot size. Significant increase in brightness can result by using lower emittance electron beams and higher power lasers.

In Table 1 an example is presented of a $90^{\circ}$ Thomson scattering source for a laser wavelength of $0.8 \mu \mathrm{m}\left(\mathrm{Ti}: \mathrm{Al}_{2} \mathrm{O}_{3}\right.$ laser) and Table 2 presents an example at $10 \mu \mathrm{m}\left(\mathrm{CO}_{2}\right.$ laser $)$ using $180^{\circ}$ Thomson scattering. In both tables, $K_{L}^{2}<1$ 
and the x-ray photon energy has been fixed at $12.4 \mathrm{keV}$. The collection angle for the flux is assumed to be $1 \mathrm{mrad}$.

\subsection{Status of ps Thomson scattering source development}

At the Accelerator Test Facility of BNL, Thomson backscattering experiments are currently underway using a photocathode RF gun/linac $(60 \mathrm{MeV}, 0.5-1$ $\mathrm{nC}$ per $3.5-10 \mathrm{ps}$ bunch) and a $\mathrm{CO}_{2}$ laser $(10.6 \mu \mathrm{m}, 600 \mathrm{MW}$ per $180 \mathrm{ps}$ pulse). ${ }^{11}$ In the co-linear counterpropagating geometry, x-rays are generated at $6.5 \mathrm{keV}$. The number of x-rays between $5-6.5 \mathrm{keV}$ was found to be $5 \times 10^{6}$ photons/pulse. Using a spectral bandwidth of the source of about $15 \%$, an rms source size of $40 \mu \mathrm{m}$, an opening angle of about $1 / \gamma$, and a repetition rate of $1 \mathrm{~Hz}$, implies an average flux of $3 \times 10^{4} \mathrm{ph} / \mathrm{s} / 0.1 \% \mathrm{BW}$ and a brightness of $8.2 \times 10^{6} \mathrm{ph} / \mathrm{s} / 0.1 \% \mathrm{BW} / \mathrm{mm}^{2} \mathrm{mrad}^{2}$. Upon completion of the ongoing ATF $\mathrm{CO}_{2}$ laser upgrade to the terawatt power level and the proposed electron bunch compression, the ATF Group expects to demonstrate x-ray yields on the order of $10^{10}$ photons/pulse, which is about three orders of magnitude larger than presently achieved.

Other approaches to Thomson scattering utilize free electron lasers. At TJNL, ${ }^{12}$ x-ray pulses with estimated duration of 400 fs have recently been produced in an intracavity collision in the center of an FEL wiggler. The electron beam ( $36.7 \mathrm{MeV}, 40 \mathrm{pC}$ per 350 fs micropulse) interacted with the FEL radiation $(5.2 \mu \mathrm{m}, 10 \mathrm{~kW}$ circulating macropulse power, $5 \mathrm{~W}$ average power) to produce $5 \mathrm{keV}$ x-rays with and macropulse and average luminosity of $2.5 \times 10^{34} \mathrm{~cm}^{-2} \mathrm{~s}^{-1}$ and $4 \times 10^{32} \mathrm{~cm}^{-2} \mathrm{~s}^{-1}$, respectively. Recent results from the Vanderbilt Free-Electron Laser were reported by Carroll et al. ${ }^{13}$ Tunable, pulsed x-rays with energies ranging from $14-18 \mathrm{keV}$ were produced by Thomson scattering between the counter-propagating FEL electron beam and its own infrared beam. The number of x-rays was measured to be on the order of $4.2 \times 10^{4}$ photons/s within a half opening angle of $5 \mathrm{mrad}$. The micropulse duration was 1 ps for both the electron and infrared pulse.

\section{Femtosecond electron bunches from Colliding Pulse Injection}

In this section, we discuss a novel scheme that uses a laser-plasma based electron source for generating truly fs electron bunches. ${ }^{14-17}$ Ultrashort electron bunches are generated by using laser pulses to dephase background plasma electrons undergoing fluid oscillations in a plasma wake. Results of numerical simulations of particle orbits in prescribed plasma and laser fields will be presented and the possibility of using this electron beam source for the generation

ICFA00b: submitted to World Scientific on February 11, 2000 
of fs x-rays through Bremsstrahlung and laser scattering is examined.

The characteristic scale length of the accelerating field in a plasma-based accelerator $^{31}$ is the plasma wavelength, $\lambda_{p}[\mathrm{~m}] \simeq 3.3 \times 10^{4} n_{p}^{-1 / 2}\left[\mathrm{~cm}^{-3}\right]$, where $n_{p}$ is the plasma density. In such short wavelength accelerators (typically $\lambda_{p} \sim$ $100 \mu \mathrm{m}$ ), production of electron beams with low momentum spread and good pulse-to-pulse energy stability requires fs electron bunches to be injected with fs synchronization with respect to the plasma wake. Although conventional electron sources (photocathode or thermionic RF guns) have achieved sub-ps electron bunches, ${ }^{26-29}$ the requirements for injection into plasma-based accelerators are presently beyond the performance of these conventional electron sources. Novel schemes which rely on laser triggered injection of plasma electrons into their own plasma wake have been proposed to generate the required fs electron bunches. ${ }^{14-17,32,33}$

Recently a new optical injection scheme was proposed ${ }^{14-17}$ which uses two relatively low intensity counter-propagating laser pulses in addition to a pump laser pulse that excites the plasma wake. This colliding pulse scheme has the ability to produce fs electron bunches with low fractional energy spreads using relatively low injection pulse intensities compared to the pump pulse $\left(a_{i n j}^{2} \ll a_{\text {pump }}^{2} \sim 1\right)$. Here $a=e A / m c^{2} \simeq 8.5 \times 10^{-10} \lambda[\mu \mathrm{m}] I^{1 / 2}\left[\mathrm{~W} / \mathrm{cm}^{2}\right]$ is the normalized vector potential, $I$ is the laser pulse intensity and $\lambda$ is the laser wavelength.

The colliding pulse optical injection scheme employs three short laser pulses (shown in Fig. 1): an intense $\left(a_{0}^{2} \simeq 1\right)$ pulse (denoted by subscript 0 ) for plasma wake generation, a forward going injection pulse (subscript 1), and a backward going injection pulse (subscript 2). The frequency, wavenumber, and normalized intensity are denoted by $\omega_{i}, k_{i}$, and $a_{i}(i=0,1,2)$. Furthermore, $\omega_{1}=\omega_{0}, \omega_{2}=\omega_{0}-\Delta \omega(\Delta \omega \geq 0)$, and $\omega_{0} \gg \Delta \omega \gg \omega_{p}$ are assumed such that $k_{1}=k_{0}$, and $k_{2} \simeq-k_{0}$.

The pump pulse generates a plasma wake with phase velocity near the speed of light $\left(v_{p 0} \simeq c\right)$. When the injection pulses collide (some distance behind the pump) they generate a slow ponderomotive beat wave with a phase velocity $v_{p b} \simeq \Delta \omega / 2 k_{0}$. During the time in which the two injection pulses overlap, a two-stage acceleration process can occur, i.e., the slow beat wave injects plasma electrons into the fast wakefield for acceleration to high energies. Injection and acceleration can occur at low densities $\left(\lambda_{p} / \lambda \sim 100\right)$, thus allowing for high single-stage energy gains, with normalized injection pulse intensities of $a_{1} \sim a_{2} \sim 0.2$, i.e., two orders of magnitude less intensity than required in Refs. ${ }^{32,33}$ Furthermore, the colliding pulse concept offers detailed control of the injection process: the injection phase can be controlled via the position of the forward injection pulse, the beat phase velocity via $\Delta \omega$, 


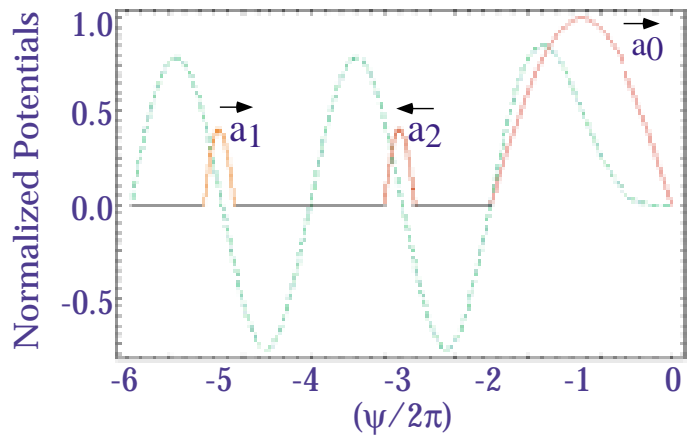

Figure 1. Normalized potential profiles of the pump laser pulse $a_{0}$, the plasma wake $\phi$, forward injection laser pulse $a_{1}$, and the backward injection laser pulse $a_{2}$.

the injection energy via the pulse amplitudes, and the injection time (number of trapped electrons) via the backward pulse duration.

\subsection{Simulations}

To evaluate the colliding pulse scheme and verify the analytical predictions, the motion of test particles in the combined wake and laser fields was simulated by numerically solving the equations of motion for the electrons using an adaptive step size Runge-Kutta method. ${ }^{16}$ Simulations of test electrons in prescribed $3 \mathrm{D}$ fields have been performed for the set of experimentally realizable parameters presented in Table 3 .

We assume the laser pulses are linear polarized fundamental Gaussian beams with half-period sine longitudinal envelopes. The polarizations of the laser pulses are chosen to be $\hat{e}_{\perp 0}=\hat{x}$ and $\hat{e}_{\perp 1}=\hat{e}_{\perp 2}=\hat{y}$ such that $\mathbf{a}_{0} \cdot \mathbf{a}_{2} \simeq 0$ and thus there is no beating (no slow wave) from the interaction of the pump pulse and the backward injection pulse. The plasma wakefields produced by the injection pulses can be neglected $\left(\phi_{1} \sim \phi_{2} \ll \phi_{0}\right)$ since the injection pulse amplitudes required for trapping are much less than the pump pulse amplitude and the pulse lengths of the injection pulses can be chosen to provide poor coupling between the plasma response and the injection pulses.

An example of injection process is given in Fig. 2, which shows the evolution in longitudinal phase space $\left(u_{z}, \psi\right)$ of the test electron distribution (a) before the collision of the injection laser pulses (in an untrapped orbit of the plasma wake) at $\omega_{p} t=37(z=240 \mu \mathrm{m})$, (b) during the collision (crossing the wake separatrix) at $\omega_{p} t=39(z=250 \mu \mathrm{m}),(\mathrm{c})$ after the collision 
Table 3. Colliding pulse Simulation parameters

$\begin{array}{ll}\text { Plasma density } n_{p} & 7 \times 10^{17} \mathrm{~cm}^{-3} \\ \text { Plasma wavelength } \lambda_{p} & 40 \mu \mathrm{m} \\ \text { Pump pulse wavelength } \lambda_{0} & 0.8 \mu \mathrm{m} \\ \text { Pump pulse length } L_{0}=\lambda_{p} & 40 \mu \mathrm{m} \\ \text { Pump pulse peak power } P & 6.7 \mathrm{TW} \\ \text { Pump laser strength } a_{0} & 0.94 \\ \text { Plasma wake potential } \phi_{o} & 0.7 \\ \text { Laser spot size } r_{s 0}=r_{s 1}=r_{s 2} & 15 \mu \mathrm{m} \\ \text { Injection laser pulse strength } a_{1}=a_{2} & 0.4 \\ \text { Injection pulse length } L_{1}=L_{2}=\lambda_{p} / 2 & 20 \mu \mathrm{m} \\ \text { Injection pulse (forward) wavelength } \lambda_{1} & 0.83 \mu \mathrm{m} \\ \text { Injection pulse (backward) wavelength } \lambda_{2} & 0.80 \mu \mathrm{m}\end{array}$

at $\omega_{p} t=50(z=320 \mu \mathrm{m})$, and (d) the resulting energetic electron bunch at $\omega_{p} t=150(z=0.95 \mathrm{~mm})$. Also shown is the $1 \mathrm{D}$ wake separatrix (dotted line). The parameters are given above except that $\hat{a}_{1}=\hat{a}_{2}=0.32$ and $L_{0}=4 L_{1}=4 L_{2}=\lambda_{p}$ with the position of the forward injection pulse centered at $\psi_{i n j}=-4 \pi$. After $z=1 \mathrm{~mm}$, Fig. 4(d), the bunch length is $1 \mathrm{fs}$ with a mean energy of $38 \mathrm{MeV}$, a fractional energy spread of $0.2 \%$, and a normalized transverse emittance of $0.9 \mathrm{~mm}$-mrad. The trapping fraction $f_{t r}$ is $3 \%$, corresponding to $2.6 \times 10^{6}$ bunch electrons. Here, $f_{t r}$ is defined as the fraction of electrons trapped that were initially loaded in a region of length $\lambda_{p} / 4$ with $r \leq 2 \mu \mathrm{m}$ (simulations indicate that electrons loaded outside this region are not trapped).

The quality of the electron bunch can be examined as the beat wave amplitude parameter $\left(\hat{a}_{1} \hat{a}_{2}\right)^{1 / 2}$ is increased beyond the threshold value for injection into a trapped and focused orbit. Shown in Fig. 3 as a function of $\left(\hat{a}_{1} \hat{a}_{2}\right)^{1 / 2}$ for $\psi_{i n j}=0$ after $0.5 \mathrm{~mm}$ of propagation are (a) the fraction of electrons that become trapped and focused (solid line), as well as the bunch duration for trapped electrons (dashed line); and (b) the fractional energy spread $\sigma_{\gamma} /\langle\gamma\rangle$ (solid line) and the transverse normalized rms emittance (dashed line) of the electron bunch. Significant trapping occurred for an injection wake phase region of $-1.5<\psi_{i n j}<1.5$. This indicates that the forward injection and pump pulses must be synchronized with an accuracy of $\sim 10 \mathrm{fs}$, which is not a serious timing constraint for present laser technology. Simulations indicate that the rms phase spread (bunch duration) is constant for a highly relativistic bunch, the fractional energy spread is asymptotic for large interaction lengths, 

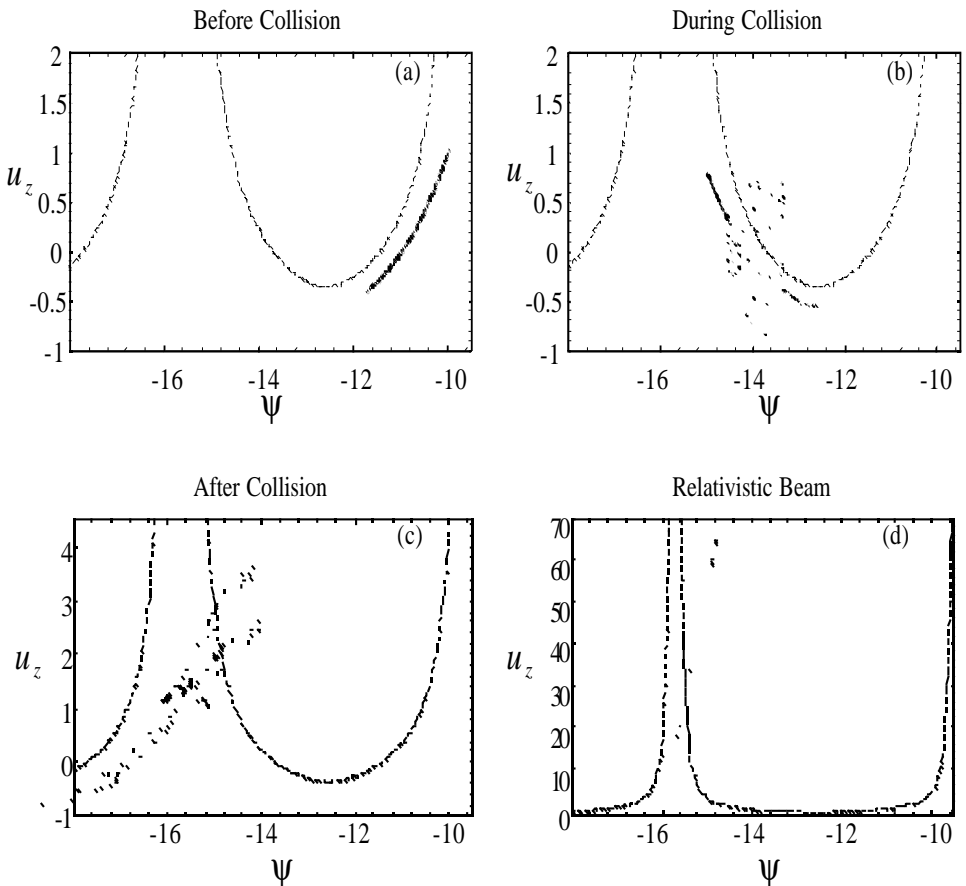

Figure 2. Electron distribution in longitudinal phase space $\left(\psi, u_{z}\right)$ (a) before the collision of injection laser pulses (in untrapped orbit of the plasma wake), (b) during the collision of injection laser pulses, (c) just after the collision, and (d) much after the collision(an energetic electron beam). The separatrix between trapped and untrapped plasma wake orbits (dotted line) is shown.

and the transverse normalized rms emittance is conserved for large pump laser spot size. As an example, for a plasma density of $N_{b}=7 \times 10^{17} \mathrm{~cm}^{-3}$, the maximum trapping fraction corresponds to a bunch number of $N_{b} \sim 0.5 \times 10^{7}$ electrons. Note that the bunch number can be increased by increasing the laser spot sizes (i.e., laser powers). For example, when the laser spot sizes are doubled to $r_{s i}=30 \mu \mathrm{m}$ in the simulation of Fig. 2 the number of trapped electrons increases to $\sim 1.5 \times 10^{7}$ and the normalized transverse emittance increases to $\sim 3.9 \mathrm{~mm}-\mathrm{mrad}$. Estimates indicate that space charge effects can be neglected while the bunch remains inside the plasma. ${ }^{16}$ 

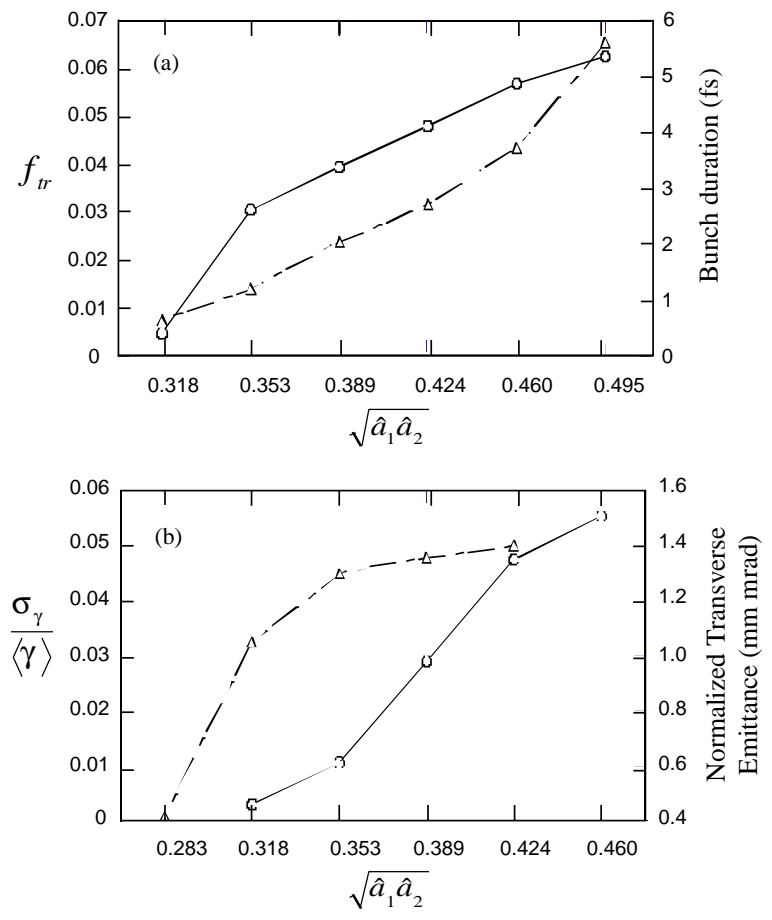

Figure 3. (a) Fraction of test electrons which become trapped and focused (solid line) and bunch duration (dashed line) versus beat wave amplitude parameter. (b) Asymptotic fractional energy spread $\sigma_{\gamma} /\langle\gamma\rangle$ (solid line) and normalized transverse rms emittance $\epsilon_{\perp}$ (mmmrad) (dashed line) of trapped electron bunch versus beat wave amplitude parameter.

\subsection{Femtosecond x-ray generation}

The fs, high current electron bunches that may be produced with the colliding pulse injector can subsequently be used to generate fs x-rays through interaction (a) with a Bremsstrahlung target or (b) with laser fields (Thomson scattering). ${ }^{34}$ The photon flux $F_{\gamma}$ at photon energy $E_{\gamma}$ produced by a relativistic electron beam with energy $E_{e}$ through Bremsstrahlung in a target with thickness $\delta$, in units of radiation lengths, is given by

$$
F_{\gamma}=N_{b} E_{e} \delta\left(\Delta E_{\gamma} / E_{\gamma}\right) / E_{\gamma}
$$

where $N_{b}$ is the number of electrons in the bunch. For a target of 0.01 radiation lengths (e.g., $35 \mu \mathrm{m}$ tungsten), the photon flux at an energy of $10 \mathrm{keV}$ is then 
Table 4. Example of a fs x-ray source (12.4 keV) for $180^{\circ}$ Thomson scattering using a $0.8 \mu \mathrm{m}$ laser and electron bunches produced through colliding pulse method. The electron bunch has a duration of $3 \mathrm{fs}$ (FWHM) which determines the duration of the x-ray pulse. The peak electron beam current is the same as for Table 1. The collection angle is assumed to be $1 \mathrm{mrad}$ and axisymmetric.

$\begin{array}{ll}\text { Laser wavelength } \lambda_{L} & 0.8 \mu \mathrm{m} \\ \text { Laser pulse energy } U_{L} & 0.5 \mathrm{~J} \\ \text { Laser pulse duration (FWHM) } \tau_{L} & 1 \mathrm{ps} \\ \text { Electron beam energy } \gamma & 50 \\ \text { Number of electrons } N_{b} & 3 \times 10^{7} \\ \text { Electron bunch length } \sigma_{b} & 1 \mu \mathrm{m} \\ \text { Normalized emittance } \epsilon_{N} & 1 \mathrm{~mm}-\mathrm{mrad} \\ \text { Bandwidth } \delta \omega / \omega & 10^{-3} \\ \text { Repetition rate } & 10 \mathrm{~Hz} \\ \text { Flux }(\mathrm{ph} / \mathrm{s} / 0.1 \% \mathrm{BW}) & 4.8 \times 10^{4} \\ \text { Brightness }\left(\mathrm{ps} / \mathrm{s} / \mathrm{mm}^{2}-\mathrm{mrad}^{2} / 0.1 \% \mathrm{BW}\right) & 1.5 \times 10^{8}\end{array}$

$6.3 \times 10^{6} E_{e}[\mathrm{MeV}]$ photons/pulse $/ 0.1 \%$ bandwidth $/ \mathrm{nC}$. The opening angle of the radiation is expected to be dominated by multiple scattering of the electrons in the target ${ }^{35}$

$$
\theta_{s c}[\mathrm{rad}]=13.6\left(\beta_{z} c p_{z}[\mathrm{MeV}]\right)^{-1} Z_{A} \sqrt{\delta}[1+0.038 \ln (\delta)]
$$

where $Z_{A}$ is the charge number of the incident particle $\left(Z_{A}=1\right.$ for an electron), and $p_{z}=\gamma \beta_{z} m c$ is the momentum of the incident particle. For a 100 $\mathrm{MeV}$ electron beam, this results in a $14 \mathrm{mrad}$ opening angle. Assuming a single bunch contains about $10^{7}-10^{8}$ electrons, Eq. (9) indicates that the total flux of x-rays produced through Bremsstrahlung with an energy of $10 \mathrm{keV}$, is on the order of $10^{6}-10^{7} \mathrm{x}$-rays $/ 0.1 \%$ bandwidth in a few fs. In principle, larger number of electrons (and hence larger x-ray fluxes) could be obtained by using larger laser spot sizes to increase the electron trapping volume.

In the case of laser Thomson scattering, the total number of scattered photons in the backscattering geometry can be estimated by the use of Eqs. (7)-(8). The parameters for this example are summarized in Table 4, assuming the electron beam parameters from simulations discussed in the previous section. From this example we conclude that this unique source, capable of producing truly fs pulses, should have sufficient flux and brightness to perform pump probe type experiments. 


\section{Conclusion}

An important aspect of the use of ultrashort pulse laser systems is that production of ultrashort pulses of x-ray radiation becomes feasible. Short x-ray pulses could have important applications in condensed matter research. To date the study of ultrafast processes has largely relied on fs optical pulses from mode-locked lasers. Since x-rays interact with core electronic levels and hence are effective structural probes, the availability of fs x-ray pulses and the intrinsic synchronization between laser and x-ray pulses would make it possible to directly probe changes in atomic structure on ultrafast time scales.

The fs Thomson scattering source, developed at LBNL, relied on the interaction of a $100 \mathrm{fs}$ long laser pulse with peak power around $0.5 \mathrm{TW}$, at $90^{0}$ with a 30 ps long electron bunch. ${ }^{7-9}$ The peak (average) flux was on the order of $6 \times 10^{2} \mathrm{ph} / \mathrm{s} / 0.1 \% \mathrm{BW}$ and the brightness was approximately 170 $\mathrm{ph} / \mathrm{s} / 0.1 \% \mathrm{BW} / \mathrm{mm}^{2}-\mathrm{mrad}^{2}$. Some of the main limitations of the experiment were the long duration of the electron bunch, the high emittance and relatively low laser power.

Experiments such as those that are underway at BNL's ATF are being designed to produce flux and brightness greater than $10^{4} \mathrm{ph} / \mathrm{s} / 0.1 \% \mathrm{BW}$ and $5 \times 10^{7} \mathrm{ph} / \mathrm{s} / 0.1 \% \mathrm{BW} / \mathrm{mm}^{2}-\mathrm{mrad}^{2}$, respectively. ${ }^{11}$ These experiments are relying on the use of shorter electron bunches obtained from state-of-the-art photocathode electron guns.

The colliding pulse optical injection scheme has the ability to generate ultrashort electron bunches by colliding laser pulses to dephase background plasma electrons undergoing fluid oscillations in a plasma wake. ${ }^{14-17} \mathrm{Sim}-$ ulations indicate fs electron bunches with moderate charge (1-10 pC), low fractional energy spread $(<1 \%)$ and low normalized transverse emittance $(\sim 1 \mathrm{~mm} \mathrm{mrad})$ can be produced. The colliding pulse scheme requires relatively low laser power compared to the pump pulse $a_{1}^{2} \sim a_{2}^{2} \ll a_{0}^{2}$, and allows for detailed control of injection process through the injection phase (position of the forward injection laser pulse), beat wave velocity (frequencies of the injection laser pulses), and the beat wave amplitude parameter (injection pulse intensities). Such an electron beam would permit generation of ultra-short x-ray pulses with truly fs duration and with flux and brightness comparable to state-of-the-art sources based on long pulses.

Another possibility for producing ultra-short electron bunches (and subsequently x-ray pulses) is a storage ring based concept know as beam slicing, ${ }^{36}$ which has recently been demonstrated at LBNL. ${ }^{37}$ In these experiments, a short pulse laser co-propagating with a relativistic electron beam $(1.9 \mathrm{GeV})$, caused a timeslice of the electron beam, with a duration approximately equal

ICFA00b: submitted to World Scientific on February 11, 2000 
to the laser pulse, to be energy modulated by up to $9 \mathrm{MeV}$ via the FEL interaction. Through the use of a dispersive section, spatial separation between the main bunch and these slices is achieved, allowing generation of fs x-ray pulses. It should be noted that the main difference in flux and brightness between the Thomson scattering sources and the optical slicing methods is that the former produces higher peak flux and brightness but that the latter produces more pulses per second.

Laser-based x-rays sources offer unique properties. For all such sources, however, laser repetition rate and average power have been one of the main limitations. Future research into the development of high average power lasers, as well as optical storage cavities for ultra-short pulses, would have a tremendous impact in the scientific reach of these electron beam/laser-based sources.

\section{Acknowledgments}

The authors acknowledge useful conversations with C.B. Schroeder, S. Chattopadhyay, R.W. Schoenlein, J.S. Wurtele, and M. Zolotorev. This work was supported by the Department of Energy, Contract No. DE-AC-03-76SF0098.

\section{References}

1. C. Rischel et al., Nature (London) 390, 490 (1997).

2. C.V. Shank et al., Phys. Rev. Lett. 50, 454 (1983); 51 ,900 (1983); L. Huang et al., Phys. Rev. Lett. 80, 185 (1998); I.L. Shumay and U. Hofer, Phys. Rev. B 53, 15878 (1996).

3. P. Sprangle, A. Ting, E. Esarey and A. Fisher, J. Appl. Phys. 72, 5032 (1992); P. Sprangle and E. Esarey, Phys. Fluids B 4, 2241 (1992).

4. E. Esarey, S.K. Ride and P. Sprangle, Phys. Rev. E 48, 3003 (1993).

5. K.J. Kim et al., Nucl. Instr. Meth. A 341, 351 (1994).

6. S.K. Ride, E Esarey and M. Baine. Phys. Rev. E 52, 5425 (1995); E. Esarey et al., Nucl. Instr. Meth. A 375, 545 (1996).

7. R.W. Schoenlein et al., Science 274, 236 (1996).

8. W.P. Leemans et al., Phys. Rev. Lett. 77, 4182 (1996).

9. W.P. Leemans et al., IEEE J. Quantum Electron. 33, 1925 (1997).

10. A. H. Chin et al., Phys. Rev. Lett. 83, 336 (1999).

11. S. Kashiwagi et al., New Visions in Laser-Beam Interactions, Tokyo Metropolitan Univ., Oct 11-15, 1999; BNL Report 66934.

12. G. Krafft, private communication (1999).

13. F.E. Carroll et al., SPIE Proc. 3614, 139 (1999).

14. E. Esarey et al., Phys. Rev. Lett. 79, 2682 (1997). 
15. W. P. Leemans et al., Phys. Plasmas 5, 1615 (1998).

16. C.B. Schroeder et al., Phys. Rev. E 59, 6037 (1999).

17. E. Esarey et al., Phys. Plasmas 6, 2262 (1999).

18. F.R. Arutyumian and V.A. Tumanian, Phys. Lett. 4, 173 (1963).

19. R.H. Milburn, Phys. Rev. Lett. 10, 75 (1963).

20. G. Fiocco and E. Thompson, Phys. Rev. Lett. 10, 89 (1963).

21. M.D. Perry and G. Mourou, Science 264, 917-24 (1994).

22. See for example http://www.fel.duke.edu/

23. T. Shintake, Nucl. Instr. Methods A 311, 453 (1992).

24. V. Balakin et al., Phys. Rev. Lett. 74, 2479-82 (1995).

25. A. Ting et al., private communication.

26. P. Kung et al., Phys. Rev. Lett. 73, 967 (1994).

27. M. Uesaka et al., Phys. Rev. E 50, 3068-76 (1994).

28. B. E. Carlsten and S. J. Russell, Phys. Rev. E 53, 2072 (1996).

29. X. J. Wang, X. Qui, and I. Ben-Zvi, Phys. Rev. E 54, 3121 (1996).

30. W.P. Leemans et al., Proc. 1993 Particle Accelerator Conf., 83 (1993).

31. E. Esarey et al., IEEE Trans. Plasma Sci. PS-24, 252 (1996).

32. D. Umstadter et al., Phys. Rev. Lett. 76, 2073 (1996).

33. R. G. Hemker et al., Phys. Rev. E 57, 5920 (1998).

34. W. P. Leemans et al., SPIE Conf. Proc. 3451, 41-50 (1998).

35. Review of Particle Physics, European Phys. J. C 3, 144 (1998).

36. A. A. Zholents and M. S. Zolotorev, Phys. Rev. Lett. 76, 912 (1996).

37. R.W. Schoenlein et al., submitted to Science (1999). 